\title{
BMJ Open Impact of the COVID-19 pandemic on vaccine coverage for early childhood vaccines in Alberta, Canada: a population-based retrospective cohort study
}

\author{
Shannon E MacDonald (D) ,,2 Yuba Raj Paudel (D) ,,2 Marilou Kiely, ${ }^{3,4}$ \\ Ellen Rafferty, ${ }^{2,5}$ Manish Sadarangani, ${ }^{6,7}$ Joan L Robinson (1) ,8 \\ S Michelle Driedger, ${ }^{9}$ Lawrence W Svenson (D),${ }^{10,11}$ on behalf of the COVImm \\ study team
}

To cite: MacDonald SE, Paudel YR, Kiely M, et al. Impact of the COVID-19 pandemic on vaccine coverage for early childhood vaccines in Alberta, Canada: a population-based retrospective cohort study. BMJ Open 2022;12:e055968. doi:10.1136/ bmjopen-2021-055968

- Prepublication history and additional supplemental material for this paper are available online. To view these files, please visit the journal online (http://dx.doi.org/10.1136/ bmjopen-2021-055968).

Received 29 July 2021 Accepted 22 December 2021

Check for updates

(c) Author(s) (or their employer(s)) 2022. Re-use permitted under CC BY-NC. No commercial re-use. See rights and permissions. Published by BMJ.

For numbered affiliations see end of article.

Correspondence to Dr Shannon E MacDonald; smacdon@ualberta.ca

\section{ABSTRACT}

Objective To assess the impact of the COVID-19 pandemic on early childhood vaccination coverage in Alberta, Canada.

Setting Alberta, a western Canadian province, which has a population of 4.4 million and approximately 50000 births annually.

Design In this retrospective cohort study, populationbased administrative health data were analysed to determine the vaccination coverage for measlescontaining, pertussis-containing and rotavirus vaccines.

Primary outcome measure We measured monthly and cumulative vaccine coverage. We assessed the absolute difference in monthly and cumulative coverage for each vaccine dose by comparing children due for vaccination in each month of 2019 and 2020, with follow-up to determine if missed doses were caught up later. Participants We included 114178 children in the 2019 analysis cohort and 106530 children in the 2020 analysis cohort.

Results Monthly vaccination coverage in 2020 was higher than 2019 until March, when coverage significantly declined. Comparing April 2020 to 2019, coverage was $9.9 \%(95 \% \mathrm{Cl} 7.9 \%$ to $12.0 \%)$ lower for measles vaccine; $4.9 \%(95 \% \mathrm{Cl} 3.3 \%$ to $6.5 \%), 7.1 \%$ (95\% Cl $5.2 \%$ to $9.1 \%), 5.2 \%(95 \% \mathrm{Cl} 3.1 \%$ to $7.4 \%)$ and $8.8 \%(95 \% \mathrm{Cl}$ $6.6 \%$ to $10.9 \%$ ) lower for first, second, third and fourth doses of pertussis-containing vaccine, respectively; and $4.0 \%$ (95\% Cl 2.3\% to $5.7 \%$ ), $7.1 \%$ (95\% Cl $5.1 \%$ to 9.2\%) and $4.6 \%$ (95\% Cl 2.4\% to $6.7 \%$ ) lower for first, second and third doses of rotavirus vaccine, respectively. Monthly coverage improved during May to July 2020; however, some doses experienced a second decline during September to October 2020. The cumulative coverage analysis showed that the measles-containing vaccine had the largest difference in coverage at the end of follow-up.

Conclusions Children who were due for vaccination early in the pandemic and in Fall 2020, especially those due for measles vaccination, may require additional catch-up.

\section{Strengths and limitations of this study}

- This is one of the few studies in Canada assessing the impact of the COVID-19 pandemic on childhood vaccine coverage.

- We used a population-based administrative database which has complete and timely data on immunisation.

- The findings from this study are based on Albertan children and may not be generalisable to other jurisdictions.

\section{INTRODUCTION \\ Background}

Health systems worldwide have struggled to ensure access to routine vaccination during the COVID-19 pandemic. Implementation of physical distancing measures and reassignment of public health workers to COVID-19 screening efforts have led to disruptions in routine healthcare, ${ }^{1-4}$ while fear of contracting COVID-19 when accessing health services has also contributed to reduced service utilisation. ${ }^{5}$ Immediate declines in vaccination uptake due to the pandemic have been previously reported, but long-term follow-up is lacking and data from Canada are limited. ${ }^{1246}$

Following the detection of the first confirmed case of COVID-19 in Alberta on 5 March 2020, the Alberta government implemented various measures to reduce virus transmission. ${ }^{7}$ These measures changed over time, varying in how restrictive they were in response to disease epidemiology (online supplemental appendix figure A1). In line with national recommendations, ${ }^{8} 9$ Alberta prioritised routine early 
childhood vaccinations, with some adjustments in service delivery (eg, vaccination-only appointments, COVID-19 screening, physical distancing). ${ }^{10}$

It is imperative to quantify the impact of COVID-19 on routine vaccination to aid public health in identifying vaccines with particularly low coverage. This will support the development of strategies for catching up coverage to prepandemic levels to reduce the risk of future outbreaks once pandemic restrictions are removed. Additionally, studying the impact of the COVID-19 pandemic on routine immunisations in various jurisdictions provides clinicians and policy makers with information on the impact of variable strategies for maintaining continuity of vaccine programmes in different contexts, which can aid in planning for related challenges that we will likely encounter in the future.

As such, the objectives of this study were (1) to evaluate vaccine coverage in Alberta for selected early childhood vaccines in 2020 and to compare it with the coverage in 2019; and (2) to investigate whether children who were delayed in their vaccinations at the start of the pandemic were caught up by March 2021.

\section{METHODS}

\section{Design}

A retrospective cohort design was used to assess early childhood vaccine coverage before (2019) and during the pandemic (2020). We used the Strengthening the Reporting of Observational Studies in Epidemiology cohort checklist when writing our report. ${ }^{11}$

\section{Setting}

Alberta is a large western Canadian province with an estimated population of 4.4 million $^{12}$ and approximately 50000 births annually. ${ }^{13}$ Almost 99\% of Albertans are registered under the universal, publicly funded Alberta Health Care Insurance Plan (AHCIP), which covers all vaccines listed in the Alberta Ministry of Health $(\mathrm{MoH})$ routine vaccine schedule. Routine vaccines for infant and preschool-age children in Alberta are exclusively administered by public health nurses in community-based clinics. $^{14}$

\section{Data}

The pandemic and prepandemic cohorts were created by linking population-based administrative data from the $\mathrm{MoH}$, including vital statistics, birth registrations and AHCIP data. Vaccination status was determined from the Immunization and Adverse Reaction to Immunization (Imm/ARI) repository, which includes information on all publicly funded childhood vaccines administered in Alberta with some exceptions, described below. ${ }^{15}$ All children born in Alberta were included in the analysis unless they died or migrated out of Alberta before their eligible date for vaccination, identified as First Nations (as data are not consistently submitted to Imm/ARI), or were from Lloydminster (as vaccines are delivered by a neighbouring province of Saskatchewan). Vaccine doses received outside the minimum or maximum age, or minimum interval between two doses according to the schedule (see online supplemental appendix table A1), were also excluded.

\section{Vaccination schedule}

This study examined vaccine coverage for three early childhood vaccines, each with unique scheduling and dose requirements (online supplemental appendix table A1). These included pertussis-containing vaccines, namely diphtheria-tetanus-acellular pertussis-polio-Haemophilus influenzae type b-hepatitis B (DTaP-IPV-Hib-HB) and DTaP-IPV-Hib; measles-mumps-rubella-varicella (MMRV) vaccine, which is sometimes administered as separate measles-mumps-rubella (MMR) and varicella vaccines; and rotavirus vaccine. We compared vaccination coverage in 2019 and 2020, during which there were no changes in the vaccination schedule.

\section{Measures}

Vaccine coverage was defined as the proportion of ageeligible children who were vaccinated by 30 or 31 days following the due date (28 or 29 days following February due dates). Online supplemental appendix table A2 shows the number of eligible children for each vaccine dose. The impact of the COVID-19 pandemic on coverage was examined in two ways: monthly coverage and cumulative coverage.

\section{Monthly coverage}

We assessed monthly coverage for each vaccine dose from January through December 2020. Coverage during each month in 2020 was compared with the corresponding month in 2019. Each month was analysed as a separate cohort. For example, the April 2020 cohort for the first dose of rotavirus vaccine consisted of children who turned 3 months old in April 2020 (ie, were due for vaccination in March 2020 when they were 2 months old). The comparison cohort was children who turned 3 months old in April 2019. This was repeated for all other months and vaccine doses.

\section{Cumulative coverage}

We assessed cumulative coverage to investigate whether children who had delayed vaccinations due to the pandemic were caught up later. Specifically, we followed the February to May 2020 cohorts until March 2021 and followed the February to May 2019 cohorts until March 2020 to estimate change in coverage levels over time. Cumulative coverage for the first and third doses of rotavirus vaccine was not calculated due to the short time window when these doses can be given. ${ }^{16}$ Follow-up data for the second dose of rotavirus vaccine were only available for children up to 8 months old.

\section{Statistical analyses}

Statistical analyses were carried out in April to May 2021 using SAS V.9.4 (SAS Institute) and Microsoft Excel. 


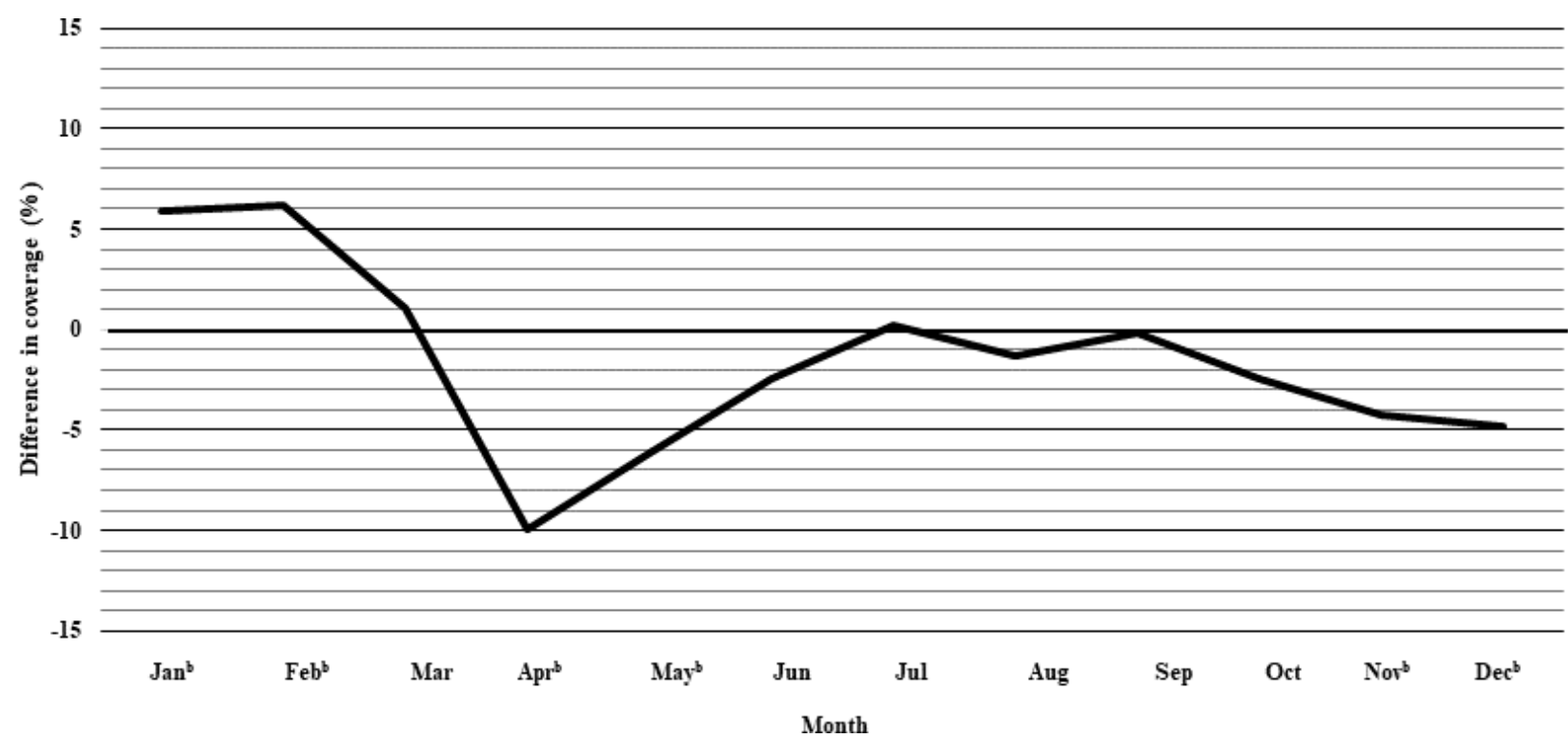

Figure 1 Difference in monthly coverage (2020 minus 2019), measured at 1 month following due date, ${ }^{a}$ for MMR/MMRV vaccine among children who turned 13 months in each month, in Alberta, Canada. ${ }^{a}$ One month following due date is defined as: up to and including 30 or 31 days following the due date, depending on the calendar month (28 or 29 days for the month of February, depending on year). ${ }^{b} 95 \%$ Cls for coverage do not overlap. MMR, measles-mumps-rubella; MMRV, measles-mumpsrubella-varicella.

Differences in monthly vaccine coverage (2020 minus 2019) were calculated to compare the coverage between two periods. We calculated asymptotic $95 \%$ CIs for binomial proportions for both coverage and difference in coverage. The overlap of $95 \%$ CIs was examined to determine whether coverage differences were statistically significant. Given that we used 2019 as our comparison year, we considered the possibility that coverage for that year might have been abnormally high or low, thus accentuating or masking the impact of the pandemic. To evaluate this possibility, we examined 5-year trends in coverage for the vaccines in our study and also included coverage in January and February 2020 (ie, prepandemic) in our main analysis.

\section{Patient and public involvement}

Patients and the public were not involved in the design, conduct and dissemination of this research.

\section{RESULTS}

After exclusions, the final sample consisted of children born between June 2017 and September 2019 (n=114178) in the 2019 cohorts and children born between June 2018 and September $2020(n=106530)$ in the 2020 cohorts.

\section{Monthly coverage}

Figures 1-3 present the difference in coverage for the three vaccines, while detailed information on monthly coverage levels is presented in the online supplemental appendix tables A3-A5.

\section{MMR/MMRV vaccine}

As shown in figure 1 and online supplemental appendix table A3, vaccine coverage at 1 month past the due date for
MMR/MMRV vaccine was $5.9 \%$ (95\% CI $3.7 \%$ to $8.1 \%$ ) and $6.2 \%$ (95\% CI $4.1 \%$ to $8.3 \%$ ) higher in January and February 2020 than 2019, respectively, and equivalent in March. A large decline in coverage was observed in April and May 2020, 9.9\% (95\% CI 7.9\% to $12.0 \%$ ) and $6.2 \%$ (95\% CI $4.1 \%$ to $8.3 \%$ ) lower, respectively, than the corresponding months in 2019. Vaccine coverage recovered in June and July 2020, reaching equivalent coverage to 2019 by July and remained similar over the next few months. A second decline in vaccine coverage occurred in October 2020, and coverage remained below 2019 levels through to December.

\section{Pertussis-containing vaccine}

Pertussis-containing vaccine coverage from January to March 2020 was similar to 2019 levels for the first and second doses (figure 2 and online supplemental appendix table A4). Notably, coverage varied across doses. Fourth dose coverage was $6.3 \%$ (95\% CI $4.1 \%$ to $8.4 \%$ ) and $4.8 \%$ (95\% CI $2.7 \%$ to $6.9 \%$ ) higher in January and February 2020, respectively. Coverage for all doses dropped in April, with the second and fourth doses having the largest drops in coverage $(7.1 \%$ (95\% CI $5.2 \%$ to $9.1 \%)$ and $8.8 \%$ (95\% CI $6.6 \%$ to $10.9 \%$ ), respectively. By May, third dose coverage was $3.3 \%$ (95\% CI $1.2 \%$ to $5.5 \%$ ) higher in 2020 than 2019, but other doses remained below 2019 levels. Coverage levels for all doses were equivalent to or slightly below 2019 levels from June to August. In September 2020, there was a sharp decline in coverage of $12.9 \%$ (95\% CI $10.6 \%$ to $15.2 \%$ ) for the fourth dose, and coverage remained below 2019 levels through December 2020. For the other doses, 2020 coverage remained equivalent to or slightly below 2019 between September and December, except for the third dose, where coverage was $3.7 \%$ (95\% CI $1.6 \%$ to $5.9 \%$ ) and $2.2 \%$ (95\% 


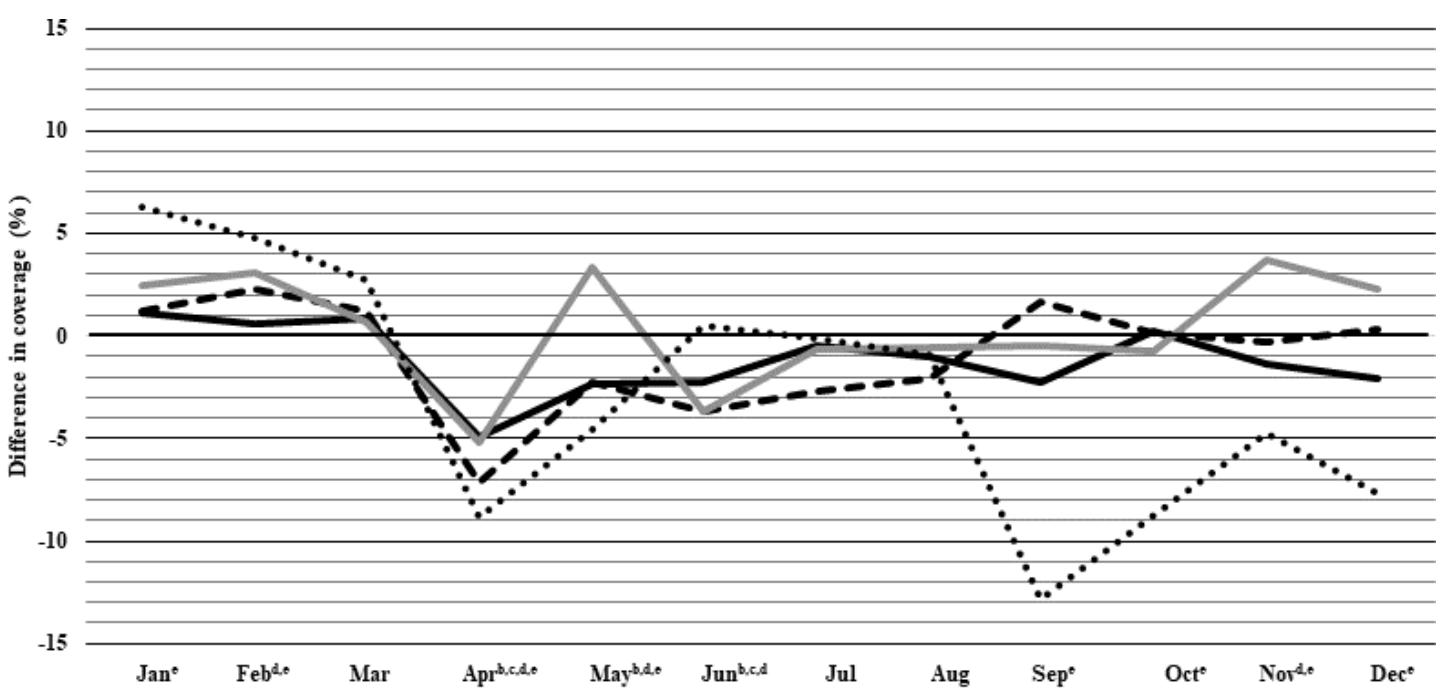

Figure 2 Difference in monthly coverage (2020 minus 2019), measured at 1 month following due date, ${ }^{a}$ for pertussis-containing vaccines among children who turned 3, 5, 7 and 19 months in each month in Alberta, Canada. ${ }^{a}$ One month following due date is defined as: up to and including 30 or 31 days following the due date, depending on the calendar month (28 or 29 for the month of February, depending on year). ${ }^{\mathrm{b}} 95 \% \mathrm{Cls}$ for coverage do not overlap for first dose. ${ }^{\mathrm{c}} 95 \% \mathrm{Cls}$ for coverage do not overlap for second dose. ${ }^{d} 95 \%$ Cls for coverage do not overlap for third dose. ${ }^{e} 95 \%$ Cls for coverage do not overlap for fourth dose.

CI $0.1 \%$ to $4.3 \%$ ) higher in November and December 2020, respectively.

\section{Rotavirus vaccine}

Rotavirus vaccine coverage in 2020 was higher than 2019 levels during January to March, with varying coverage across doses (figure 3 and online supplemental appendix table A5). The third dose had the largest difference in coverage at $2.6 \%$ (95\% CI $0.4 \%$ to $4.8 \%$ ) and $3.6 \%(95 \%$ CI $1.5 \%$ to $5.6 \%$ ) higher during January and February 2020, respectively. Coverage for all doses declined in April 2020, with the second dose having the largest drop of $7.1 \%$ (95\% CI $5.1 \%$ to $9.2 \%$ ) compared with 2019 . By May 2020, third dose coverage was 3.7\% (95\% CI 1.5\% to $5.8 \%$ ) higher than 2019 levels, but first and second

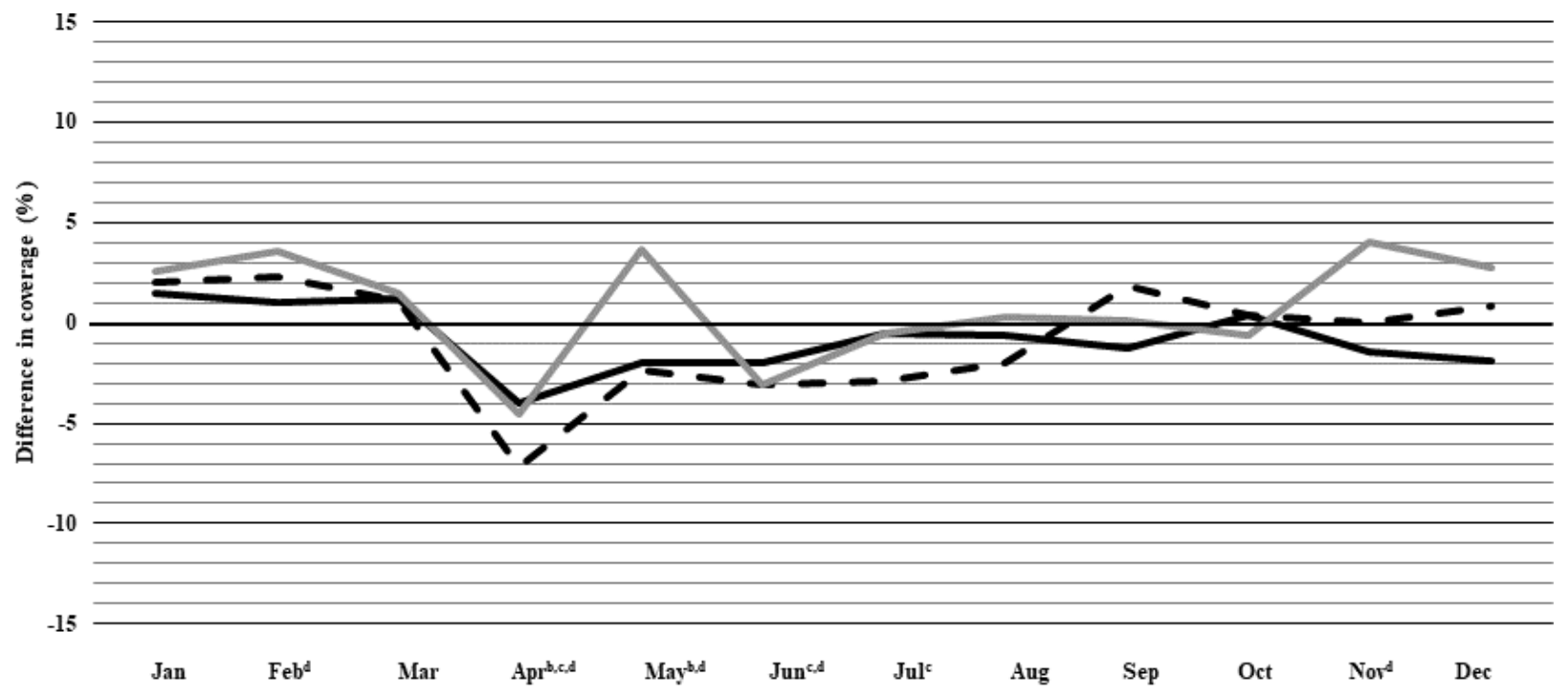

- lst dose - -2nd dose $\longrightarrow$ 3rd dose

Figure 3 Difference in monthly coverage (2020 minus 2019), measured at 1 month following due date, ${ }^{a}$ for rotavirus vaccine among children who turned 3, 5 and 7 months in each month in Alberta, Canada. ${ }^{\text {ane }}$ month following due date is defined as: up to and including 30 or 31 days following the due date, depending on the calendar month (28 or 29 for the month of February, depending on year). ${ }^{b} 95 \%$ Cls for coverage do not overlap for first dose. ${ }^{\mathrm{c}} 95 \% \mathrm{Cls}$ for coverage do not overlap for second dose. ${ }^{\mathrm{d}} 95 \% \mathrm{Cls}$ for coverage do not overlap for third dose. 

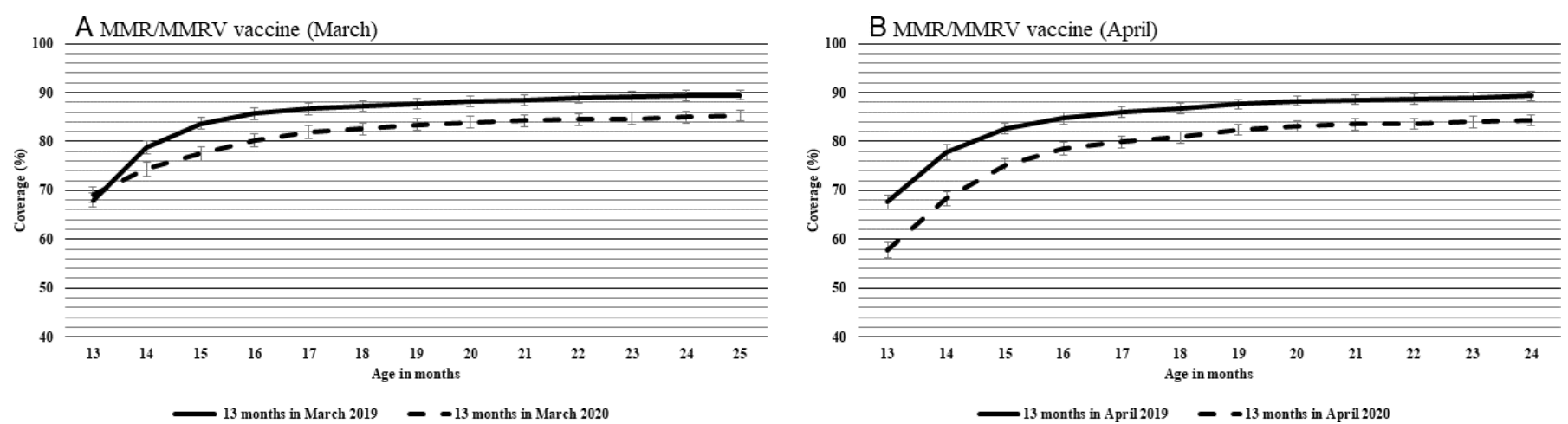

Figure 4 Cumulative coverage for MMR/MMRV vaccine for children who turned 13 months (A) in March 2019 and March 2020 and (B) in April 2019 and April 2020 in Alberta, Canada. The 2019 cohorts were followed until March 2020 and the 2020 cohorts were followed until March 2021. Bars represent 95\% Cls. MMR, measles-mumps-rubella; MMRV, measles-mumps-rubellavaricella.

dose coverage remained below 2019 levels. From June to December 2020, coverage rates for all doses were equivalent to or slightly below 2019, with the exception of the third dose, which was $4.0 \%$ (95\% CI $1.8 \%$ to $6.2 \%$ ) and $2.7 \%$ (95\% CI $0.6 \%$ to $4.9 \%$ ) higher than 2019 in November and December, respectively.

Our analysis of 5-year trends in vaccination coverage showed that coverage in 2019 was higher than in preceding years for most early childhood vaccines (online supplemental appendix figures A5-A7), but that 2020 was on track to continue that trend (see January and February 2020 coverage in figures 1-3). This validates the use of 2019 as the comparator year, and the increasing trend of vaccine coverage also explains the higher coverage in January and February 2020.

\section{Cumulative coverage}

Cumulative coverage for March and April cohorts (each followed over the following months) for each vaccine is shown below. February and May cohorts are available in the online supplemental appendix figures A2-A4.

\section{MMR/MMRV vaccine}

In the March 2020 cohort, $69 \%$ of children (who were 13 months of age) had received MMR/MMRV, similar to the March 2019 cohort (68\%) (figure 4A). The difference in coverage between the two March cohorts was larger in April 2020 (when these children were 14 months old), with the 2020 cohort having lower coverage than the 2019 cohort, and remained constant over the following months. At the end of the follow-up period (March 2020 or 2021), coverage was $85 \%$ for the March 2020 cohort compared with $90 \%$ for the 2019 cohort (figure 4A).

In the April 2020 cohorts, coverage was significantly lower in April 2020 (58\%), compared with 2019 (68\%) (figure 4B). Over the following months, the difference in coverage between the April cohorts slightly decreased. Coverage at the end of the follow-up was $84 \%$ in 2020 , compared with $89 \%$ for the 2019 cohort (figure 4B).

\section{Pertussis-containing vaccine}

Coverage for the first dose of pertussis-containing vaccines initially showed a similar pattern as observed for MMR/MMRV for both March and April cohorts (figure 5A,B). Over the following months, the difference in coverage between the March 2020 and 2019 cohorts was small and stable. At the end of follow-up, coverage was $92 \%$ for the March 2020 cohort, comparable to 93\% for the March 2019 cohort (figure 5A). For the April cohort, the difference in coverage slightly decreased during the follow-up. Coverage was $92 \%$ at the end of follow-up for the April 2020 cohort, comparable to 93\% for the April 2019 cohort (figure 5B).

Similar trends were observed for the second to fourth doses (figure 5C-H), with some differences. At the beginning of follow-up, the fourth dose had the largest difference in coverage (9\%) between the April cohorts (figure $5 \mathrm{H}$ ). The difference in coverage at the end of follow-up was also slightly higher (2\%-3\%) for the later doses of pertussis-containing vaccine compared with the first dose for both March and April cohorts.

\section{Rotavirus vaccine}

Coverage for the second dose of rotavirus vaccine at the beginning of follow-up for both March and April cohorts showed a similar pattern to MMR/MMRV and pertussis-containing vaccines (figure 6A,B). For the March and April cohorts, the difference in coverage was small and stable during follow-up. At the end of follow-up, coverage was $84 \%$ for the March 2020 cohort compared with $85 \%$ for the March 2019 cohort (figure 6A). Similarly, coverage was comparable at the end of follow-up for the April cohorts, at $83 \%$ for the 2020 cohort, compared with $85 \%$ for the 2019 cohort (figure 6B).

\section{DISCUSSION}

Monthly coverage for MMR/MMRV, pertussiscontaining and rotavirus vaccines from January to March 2020 was higher than corresponding months 


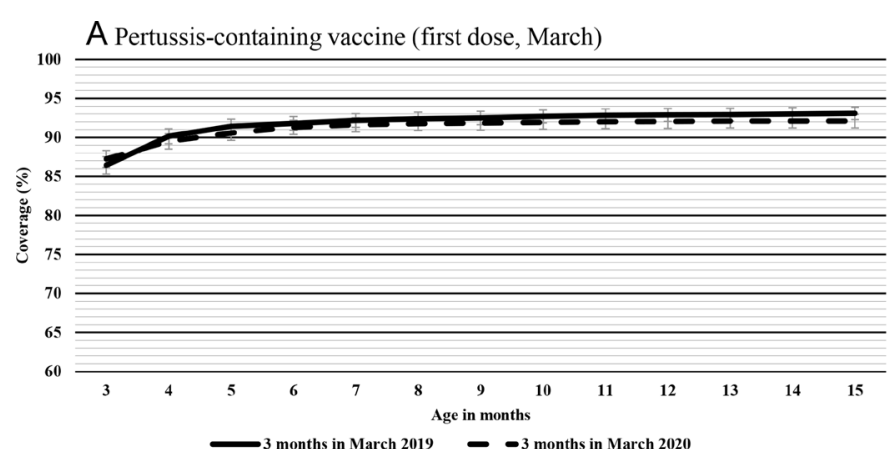

C Pertussis-containing vaccine (second dose, March)

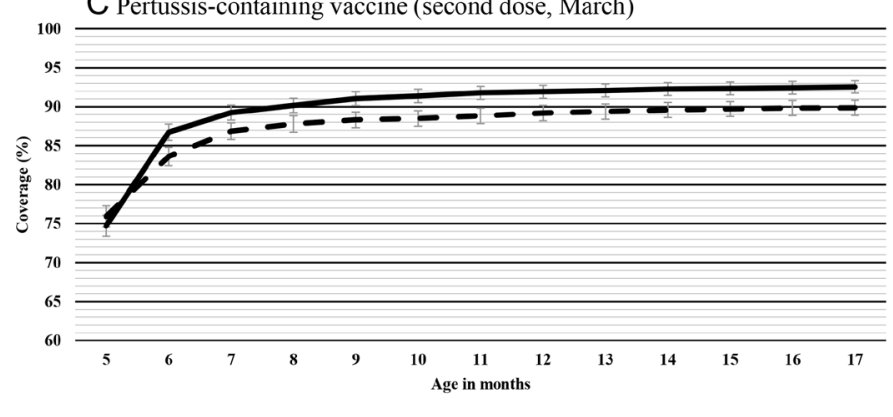

- 5 months in March $2019 \quad-\quad-5$ months in March 2020

E Pertussis-containing vaccine (third dose, March)
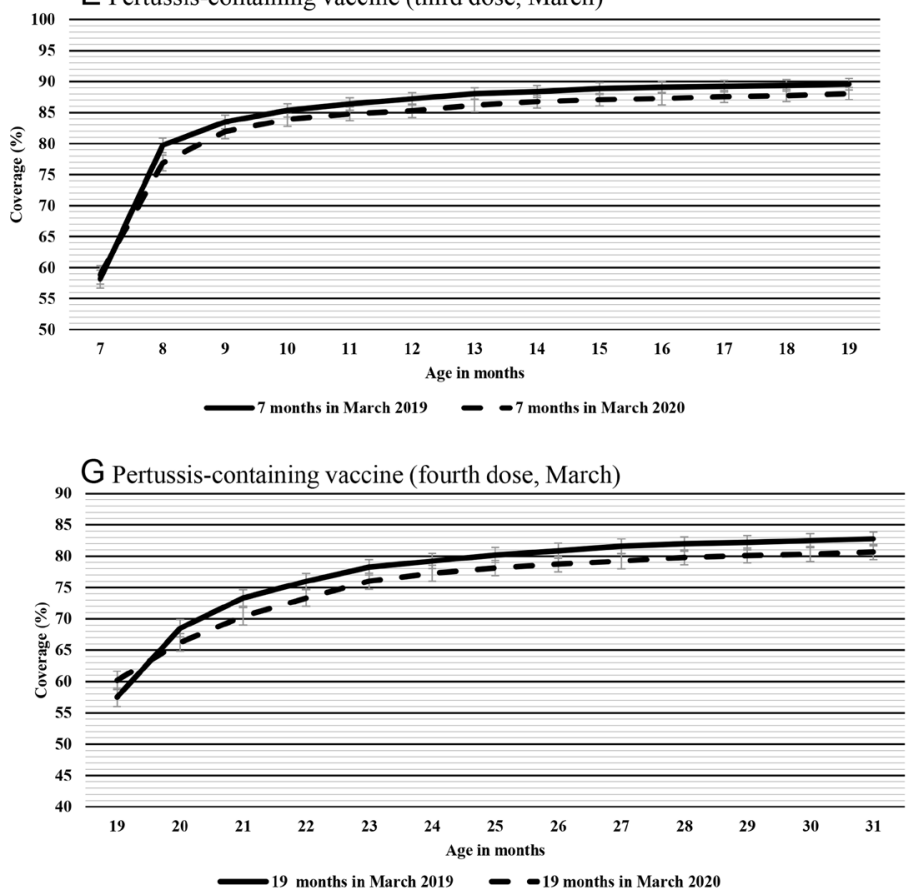

B Pertussis-containing vaccine (first dose, April)
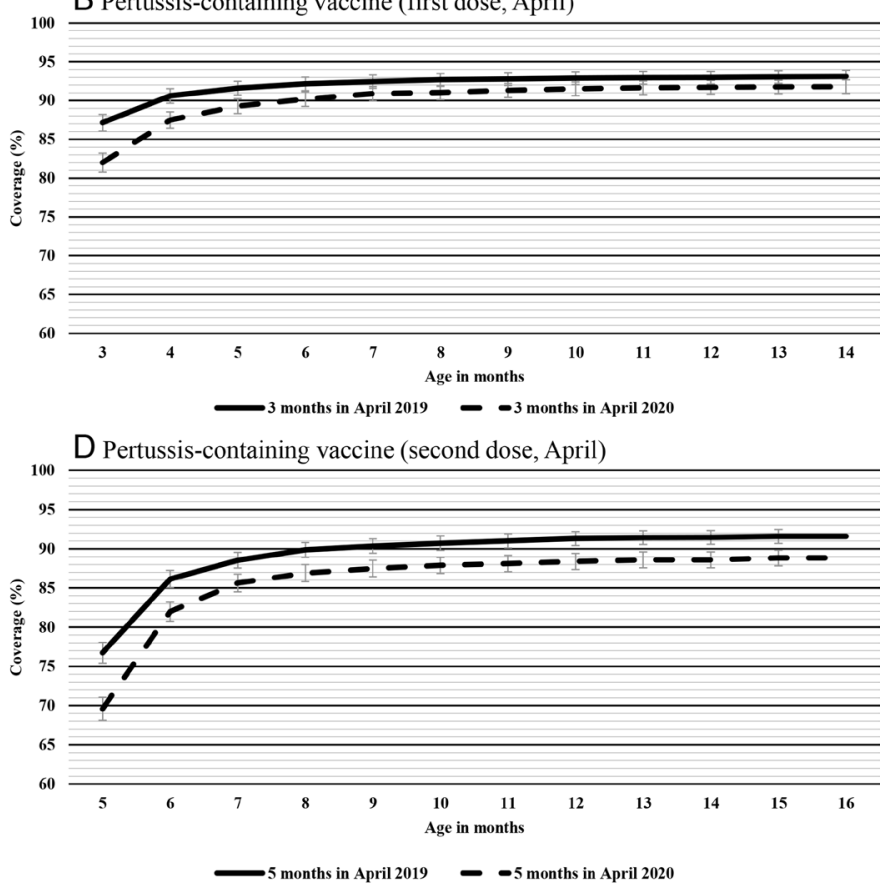

F Pertussis-containing vaccine (third dose, April)

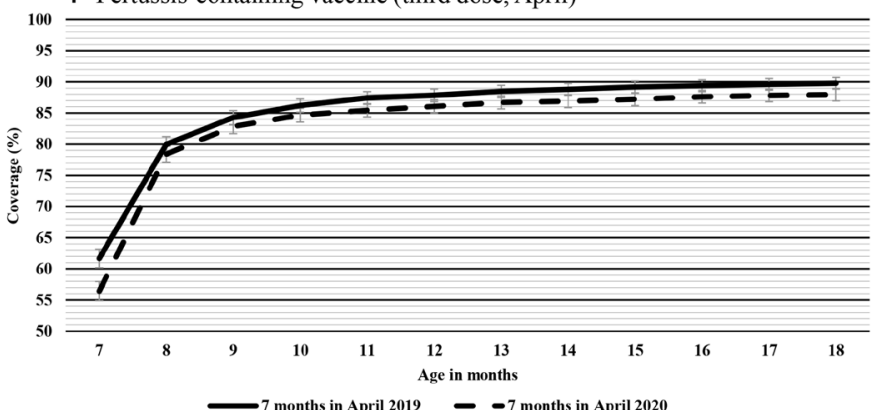

H Pertussis-containing vaccine (fourth dose, April)

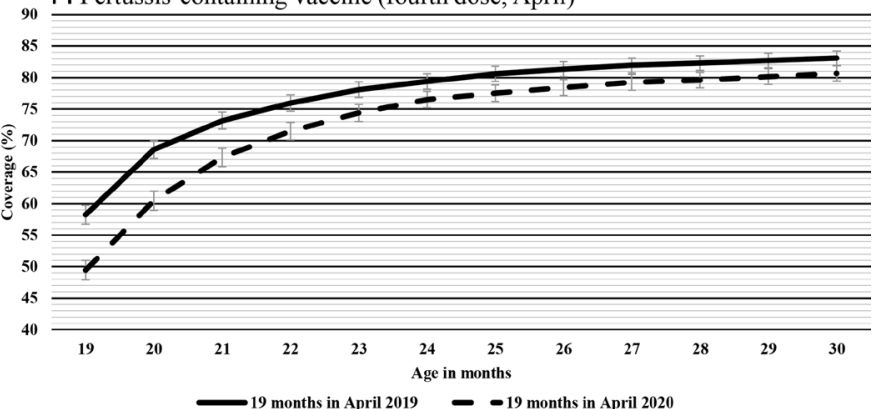

Figure 5 Cumulative coverage for the first dose of pertussis-containing vaccine for Albertan children who turned 3 months in (A) March 2019 and March 2020 and in (B) April 2019 and April 2020. Cumulative coverage for the second dose of pertussiscontaining vaccine for Albertan children who turned 5 months in (C) March 2019 and March 2020 and in (D) April 2019 and April 2020. Cumulative coverage for the third dose of pertussis-containing vaccine for Albertan children who turned 7 months in (E) March 2019 and March 2020 and in (F) April 2019 and April 2020. Cumulative coverage for the fourth dose of pertussiscontaining vaccine for Albertan children who turned 19 months in (G) March 2019 and March 2020 and in (H) April 2019 and April 2020. The 2019 and 2020 cohorts were followed until March 2020 and March 2021, respectively. Bars represent $95 \%$ Cls.

in 2019 for most of the doses. After the onset of the COVID-19 pandemic in March 2020, coverage was lower for all vaccines in April 2020 compared with 2019. However, coverage for most of the vaccines returned almost to normal levels in May and June 2020. Notably, coverage varied based on age or dose, with vaccines given at older ages showing a larger drop in 2020 compared with 2019. The exceptions were the third doses of the pertussis-containing and rotavirus vaccines, which had coverage above 2019 levels in May after declining in April. Coverage remained similar to 2019 levels throughout the summer months and early 

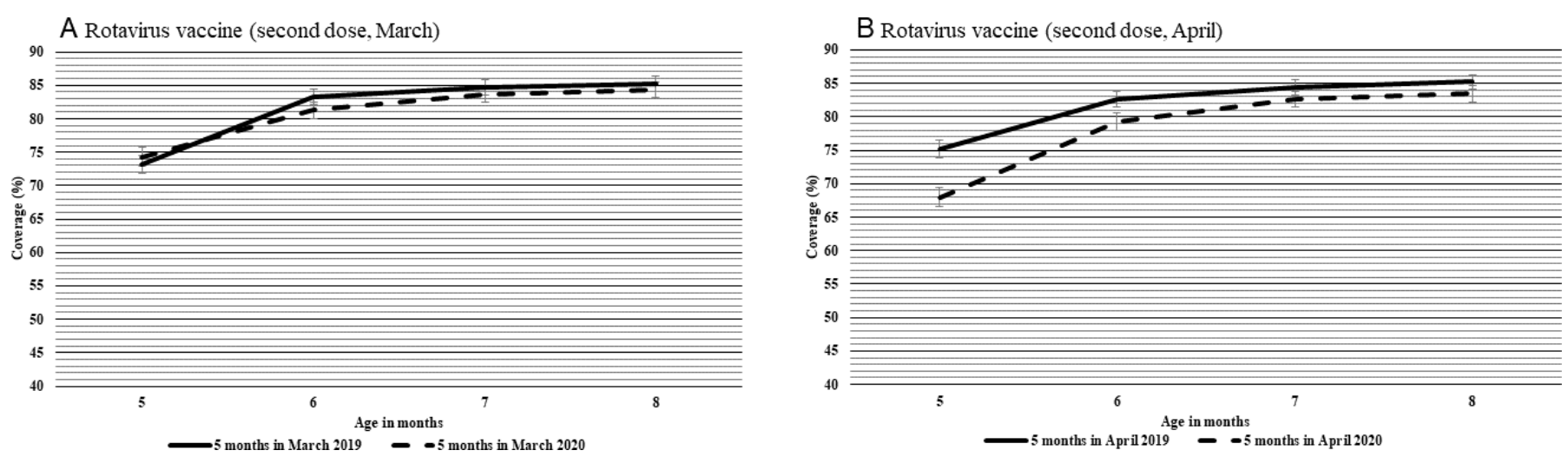

Figure 6 Cumulative coverage for rotavirus vaccine (second dose) for Albertan children who turned 5 months in (A) March 2019 and March 2020 and in (B) April 2019 and April 2020. Cohorts were followed until children turned eight months old (i.e., June 2019 or June 2020 for March cohorts, July 2019 or July 2020 for April cohorts). Bars represent 95\% Cls. Follow-up data were only available until children turned eight months old.

fall. Some vaccines experienced a second decline in coverage in the later fall months.

Cumulative coverage for the three vaccines was lower for the April 2020 cohort of children compared with April 2019 during the early stages of the pandemic but increased to levels similar to the 2019 cohort over time. The March 2020 and March 2019 cohorts had relatively similar vaccination coverage for all three vaccines throughout the pandemic. The MMR/MMRV vaccine had the highest difference in coverage comparing 2019 and 2020 at the end of the follow-up period.

Beginning in late March 2020, community health centres instituted various public health measures for vaccination appointments such as shorter appointments for infant immunisations, implementation of public health restrictions (ie, wearing personal protective equipment and screening for COVID-19) and temporary suspension of school immunisations. ${ }^{410}$ At the same time, parents' ability or willingness to access routine vaccination for their children may have been influenced by fear of contracting COVID-19. ${ }^{9}$ This likely explains the decline in early childhood vaccination coverage seen in April 2020. Some vaccines also showed a second decline in the fall, which is when additional public health restrictions were implemented in response to increased COVID-19 infections. Our finding that early childhood vaccine coverage declined after the onset of the COVID-19 pandemic aligns with literature from other jurisdictions, ${ }^{1} 2618$ although the declines in Alberta were not as extreme. In the USA, Langdon-Embry et at found a $62 \%$ decrease in infant immunisation during March and April 2020 in New York City, while Bramer et $a l^{18}$ reported declines ranging from $5 \%$ to $20 \%$ in infant vaccine coverage in May 2020 in Michigan. One possible reason for the smaller impact in Alberta is that childhood vaccinations are provided entirely in community health centres in Alberta, so the move to virtual appointments by many physicians' offices ${ }^{19} 20$ would have no impact on vaccine service delivery.
For some of the vaccine doses given at older ages (ie, 6 months or older), 2020 coverage rates were substantially lower than for younger children. Similar findings were reported in Michigan. ${ }^{18}$ One of the reasons for the reduced impact of the pandemic on doses given to infants compared with toddlers may be the higher priority given to primary immunisation series for infants. ${ }^{910}$ The exception to this trend in our study was the third dose of pertussis-containing and rotavirus vaccines, which had higher coverage in May and again in November to December 2020. Personal communication with public health managers revealed that after initial reduction of vaccine service delivery at the start of the pandemic, many community health centres were contacting parents of 6-month-old children to get them vaccinated before they aged out of rotavirus vaccine eligibility (online supplemental appendix table A1). This might also explain the increase in November to December, as increased restrictions in October may have been followed by heightened efforts to bring in children for the third dose in time.

Our findings also showed that the coverage for most of the early childhood vaccines began to recover in May and June 2020, a pattern seen elsewhere. ${ }^{6}$ It is likely that strategies such as encouraging health workers to order and provide routine vaccines, using reminders and recall tools and highlighting the importance of vaccination in public press conferences aided in the recovery of vaccination rates. ${ }^{910}$

It is noteworthy that coverage for many early childhood vaccines remained slightly below 2019 levels throughout late 2020 and early 2021. Accumulation of unimmunised and underimmunised children can lead to a corresponding increased risk of disease outbreaks. ${ }^{3}$ In a 2019 outbreak of 704 measles cases in the USA, $89 \%$ of cases were in unvaccinated individuals or those with unknown vaccination status. ${ }^{21}$ In a 2013 measles outbreak in Alberta, Canada, linked to a religious community from southern Alberta, of the 42 confirmed cases, all were unimmunised for measles. ${ }^{22}$ In light of the evidence of reduced measles vaccine 
coverage in our study, catch-up programmes to reach unimmunised/underimmunised children are critical. In particular, additional efforts such as identification, outreach and reminders sent to children who missed vaccines during the pandemic are needed to prevent potential outbreaks as COVID-19 restrictions ease and international travel increases.

\section{Strengths and limitations}

A strength of our study was the use of a populationbased repository that contains complete and timely information on all childhood vaccinations, as well as complete denominator data, which is an improvement over previous research that only had access to counts of children vaccinated. ${ }^{2}$ We also included data on a variety of early childhood vaccines and age groups over an extended period to examine coverage at different periods of the pandemic.

As this study focused on vaccination coverage for Albertan children, findings may not be generalisable to children in other jurisdictions. Additionally, the findings presented here do not infer causality, that is, we cannot infer that COVID-19 pandemic is the cause of decline in vaccine coverage seen in 2020 compared with 2019.

\section{Future directions}

Future research could investigate reasons for vaccination decreases, specifically through examination of the perspectives and experiences of parents, vaccination providers and programme coordinators. This information would be useful for any future pandemic preparedness planning to maintain routine vaccination coverage. Additionally, analysis of the characteristics of those who are missing vaccine doses may be helpful for the development of vaccination catch-up programmes.

\section{CONCLUSION}

Monthly coverage analyses showed that coverage for early childhood vaccines in Alberta declined in April 2020 following onset of the COVID-19 pandemic. Coverage for most of the early childhood vaccines improved in May and June 2020. Cumulative coverage analyses showed that coverage was initially lower for the April 2020 cohort in comparison to 2019 but increased to similar levels over time. The MMR/MMRV vaccine had the largest difference in coverage at the end of follow-up, when comparing 2019 and 2020, suggesting that this vaccine may be a priority for catch-up programmes.

\footnotetext{
Author affiliations

${ }^{1}$ School of Public Health, University of Alberta, Edmonton, Alberta, Canada

${ }^{2}$ Faculty of Nursing, University of Alberta, Edmonton, Alberta, Canada

${ }^{3}$ Institut national de santé publique du Québec, Quebec, Quebec, Canada

${ }^{4}$ Département de médecine sociale et préventive, Université Laval, Quebec, Quebec, Canada

${ }^{5}$ Institute of Health Economics, Edmonton, Alberta, Canada
}

${ }^{6}$ Vaccine Evaluation Center, BC Children's Hospital Research Institute, Vancouver, British Columbia, Canada

${ }^{7}$ Department of Pediatrics, The University of British Columbia, Vancouver, British Columbia, Canada

${ }^{8}$ Department of Pediatrics, University of Alberta, Edmonton, Alberta, Canada ${ }^{9}$ Department of Community Health Sciences, University of Manitoba, Winnipeg, Manitoba, Canada

${ }^{10}$ Alberta Health, Government of Alberta, Edmonton, Alberta, Canada

${ }^{11}$ Division of Preventive Medicine, University of Alberta, Edmonton, Alberta, Canada

Twitter Shannon E MacDonald @SE_MacDonald and Lawrence W Svenson @ Larry_Svenson

Acknowledgements We express thanks to Hannah Sell for her assistance with drafting the manuscript.

Collaborators This study was part of a larger project conducted by the COVImm study team, which included the named authors as well as K Benzies, J Bettinger, $\mathrm{E}$ Dubé, A Gagneur, R Humble, S Meyer, N MacDonald and S Wilson.

Contributors SEM was involved in conceptualisation, methodology, investigation, formal analysis, funding acquisition, writing (original draft, review and editing) and supervision. YRP was involved in investigation, formal analysis and writing (original draft, review and editing). MK, ER, MS, JLR, SMD and LWS assisted with conceptualisation, methodology and writing (review and editing). SEM is the guarantor who accepts full responsibility for the work.

Funding This work was supported by an operating grant from the Canadian Institutes of Health Research (grant number VR5-172700).

Disclaimer The funding source had no role in the design and conduct of the study. Competing interests MS is supported via salary awards from the BC Children's Hospital Foundation, the Canadian Child Health Clinician Scientist Program and the Michael Smith Foundation for Health Research. MS has been an investigator on projects funded by GlaxoSmithKline, Merck, Pfizer, Sanofi-Pasteur, Seqirus, Symvivo and VBI Vaccines. All funds have been paid to his institute, and he has not received any personal payments. SEM is supported by a salary award from the Canadian Child Health Clinician Scientist Program.

\section{Patient consent for publication Not required}

Ethics approval Ethical approval was obtained from the University of Alberta Health Research Ethics Board (Ethics ID: Pro00102401). A waiver of consent was obtained from ethics, as the administrative data were deidentified before being shared with the research team.

Provenance and peer review Not commissioned; externally peer reviewed.

Data availability statement № data are available. The steward of the data used in this study is the Alberta Ministry of Health, who maintains the data for the purpose of health system administration. Thus, the authors are not at liberty to make the data publicly available.

Supplemental material This content has been supplied by the author(s). It has not been vetted by BMJ Publishing Group Limited (BMJ) and may not have been peer-reviewed. Any opinions or recommendations discussed are solely those of the author(s) and are not endorsed by BMJ. BMJ disclaims all liability and responsibility arising from any reliance placed on the content. Where the content includes any translated material, BMJ does not warrant the accuracy and reliability of the translations (including but not limited to local regulations, clinical guidelines, terminology, drug names and drug dosages), and is not responsible for any error and/or omissions arising from translation and adaptation or otherwise.

Open access This is an open access article distributed in accordance with the Creative Commons Attribution Non Commercial (CC BY-NC 4.0) license, which permits others to distribute, remix, adapt, build upon this work noncommercially, and license their derivative works on different terms, provided the original work is properly cited, appropriate credit is given, any changes made indicated, and the use is non-commercial. See: http://creativecommons.org/ licenses/by-nc/4.0/.

\section{ORCID iDs}

Shannon E MacDonald http://orcid.org/0000-0003-4675-4433

Yuba Raj Paudel http://orcid.org/0000-0003-3403-4359

Joan L Robinson http://orcid.org/0000-0001-9831-5681

Lawrence W Svenson http://orcid.org/0000-0002-3391-578X 


\section{REFERENCES}

1 Santoli JM, Lindley MC, DeSilva MB, et al. Effects of the COVID-19 pandemic on routine pediatric vaccine ordering and administration United States, 2020. MMWR Morb Mortal Wkly Rep 2020;69:591-3.

2 McDonald HI, Tessier E, White JM, et al. Early impact of the coronavirus disease (COVID-19) pandemic and physical distancing measures on routine childhood vaccinations in England, January to April 2020. Euro Surveill 2020;25:1-6.

3 Zhong Y, Clapham HE, Aishworiya R, et al. Childhood vaccinations: hidden impact of COVID-19 on children in Singapore. Vaccine 2021;39:780-5.

4 Sell H, Assi A, Driedger SM, et al. Continuity of routine immunization programs in Canada during the COVID-19 pandemic. Vaccine 2021;39:5532-7.

5 Hirabayashi K. The impact of COVID-19 on routine vaccinations: reflections during world immunization week 2020, 2020. https://www. unicef.org/eap/stories/impact-covid-19-routine-vaccinations

6 Langdon-Embry M, Papadouka V, Cheng I, et al. Notes from the Field: Rebound in Routine Childhood Vaccine Administration Following Decline During the COVID-19 Pandemic - New York City, March 1-June 27, 2020. MMWR Morb Mortal Wkly Rep 2020;69:999-1001.

7 Alberta Government. COVID-19 orders and legislation, 2021. https:// www.alberta.ca/covid-19-orders-and-legislation.aspx\#jumplinks-2

8 Canadian Pediatric Society. Stick to immunization schedule during the COVID-19 pandemic, paediatricians urge, 2020. https://www.cps. $\mathrm{ca} / \mathrm{en} / \mathrm{media} /$ stick-to-immunization-schedule-during-the-covid-19pandemic

9 National Advisory Committee on Immunization. Interim guidance on continuity of immunization programs during the COVID-19 pandemic, 2020. Available: https://www.canada.ca/en/public-health/services/ immunization/national-advisory-committee-on-immunizationnaci/interim-guidance-immunization-programs-during-covid-19pandemic.html [Accessed 20 May 2021].

10 Immunize Alberta. Immunizations during the COVID-19 pandemic, 2020. Available: https://immunizealberta.ca/i-want-immunize/ immunizations-during-covid-19-pandemic [Accessed 12 May 2021].
11 von Elm E, Altman DG, Egger M, et al. The strengthening the reporting of observational studies in epidemiology (STROBE) statement. 2007;18:800-4

12 Alberta Government. Economic Dashboard, 2020. Available: https:// economicdashboard.alberta.ca/ [Accessed 18 Nov 2020].

13 Births L. Age of mother by total live births, 2020. https://open.alberta. ca/opendata/live-births-age-of-mother-by-total-live-births

14 Rafferty E, Guo X, McDonald B, et al. Measurement of coverage, compliance and determinants of uptake in a publicly funded rotavirus vaccination programme: a retrospective cohort study. BMJ Open 2019;9:e031718.

15 Wilson SE, Quach S, MacDonald SE, et al. Immunization information systems in Canada: attributes, functionality, strengths and challenges. A Canadian immunization research network study. Can J Public Health 2017;107:e575-82.

16 Alberta Health Services. Rotavirus vaccine biological page, 2021. https://www.albertahealthservices.ca/assets/info/hp/cdc/if-hp-cdcipsm-rotavirus-vaccine-biological-page.pdf

17 Ontario Ministry of Health. Guidance for immunization services during COVID-19, 2020. http://www.health.gov.on.ca/en/pro/programs/ publichealth/coronavirus/docs/Immunization_Services_during_ COVID-19_08-26-2020.pdf

18 Bramer CA, Kimmins LM, Swanson R, et al. Decline in child vaccination coverage during the COVID-19 pandemic - Michigan Care Improvement Registry, May 2016-May 2020. MMWR Morb Mortal Wkly Rep 2020;69:630-1.

19 Koonin LM, Hoots B, Tsang CA, et al. Trends in the use of telehealth during the emergence of the COVID-19 pandemic - United States, January-March 2020. MMWR Morb Mortal Wkly Rep 2020;69:1595-9.

20 Webster P. Virtual health care in the era of COVID-19. Lancet 2020;395:1180-1.

21 Patel M, Lee AD, Redd SB, et al. Increase in measles cases - United States, January 1-April 26, 2019. Am J Transplant 2019;19:2127-30.

22 Kershaw T, Suttorp V, Simmonds K, et al. Outbreak of measles in a non-immunizing population, Alberta 2013. Can Commun Dis Rep 2014;40:243-50. 